\title{
MedChemComm
}

Check for updates

Cite this: Med. Chem. Commun., 2018, 9, 1359

Received 5th April 2018,

Accepted 28th June 2018

DOI: $10.1039 / \mathrm{c} 8 \mathrm{md} 00187 \mathrm{a}$

rsc.li/medchemcomm

\section{MicroRNA-451 blockade promotes osteoblastic differentiation and skeletal anabolic effects by promoting YWHAZ-mediated RUNX2 protein stabilization}

\author{
Jieen Pan, (D)* Chenglong Huang, Gang Chen, Zhenhai Cai and Zhongwei Zhang
}

\begin{abstract}
Background: senile osteoporosis researchers are now seeking to promote osteoblastogenesis and resultant bone formation to directly counteract age-related bone loss. Targeting microRNA (miRNA) activity in adult osteoblasts may be a successful therapeutic strategy for age-related bone loss. We investigated the mechanism(s) by which miRNAs negatively regulate osteoblastogenesis and bone formation in vitro and in vivo. Methods: we performed a miRNA microarray screen followed by PCR validation in adult bone marrow-derived mesenchymal stem cells during the proliferation-to-mineralization transition to identify downregulated miRNAs, most notably miR-451. Primary human calvarial pre-osteoblasts were isolated and transfected with miR-451's agomir or antagomir for in vitro assays. Bioinformatics analysis and in vitro experiments verified YWHAZ as a miR-451 target gene. We next investigated the effects of YWHAZ knockdown on osteoblastic differentiation. To examine the effects of miR-451's antagomir in vivo, we injected ovariectomized $(\mathrm{OVX})$ or sham-operated mice with miR-451's antagomir over a period of six weeks. We isolated stromal cells from murine bone marrow on week six for further ex vivo experimentation. Results: miR-451's antagomir stimulated pre-osteoblast differentiation into a more differentiated, mineralized phenotype. This phenotype was associated with upregulated RUNX2, ALP, and COL1A1 protein expression. miR-451's antagomir derepresses YWHAZ expression, thereby enhancing RUNX2 protein stability and promoting osteoblastic differentiation. When injected in vivo, miR-451's antagomir promotes osteoblastogenesis and mineralization, reversed OVX-induced bone loss, and increased bone strength in OVX and shamoperated mice. Conclusions: miR-451 suppresses osteoblastogenesis in vitro and in vivo. miR-451 inhibition may serve as an effective anabolic therapeutic strategy in senile osteoporosis patients.
\end{abstract}

\section{Introduction}

Age-related bone loss, termed senile osteoporosis, is characterized by reduced trabecular and periosteal bone formation relative to bone resorption. ${ }^{1}$ This bone deterioration results in bone fragility and increased fracture risk, which lead to profound increases in morbidity, mortality, and socioeconomic burden. ${ }^{1,2}$ Conventionally, the clinical management of senile osteoporosis has been based on inhibiting bone resorption via anti-resorptive medications such as bisphosphonates, denosumab, and zoledronic acid. ${ }^{1,3}$ However, due to the serious side effects associated with anti-resorptive drugs (e.g., atypical femur fractures, osteonecrosis of the jaw) that discourage patient use, there is a pressing need for novel osteoporosis therapeutics. ${ }^{2}$

Department of Orthopaedics, The Second Hospital of Jiaxing, No. 1518, Huancheng Road Nanhu District, Jiaxing 314000, Zhejiang Province, P.R. China. E-mail: 15888317769@163.com
To that end, researchers are now seeking to promote osteoblastogenesis and resultant bone formation to directly counteract age-related bone loss. ${ }^{1}$ In this field, the role of microRNAs (miRNAs) in regulating adult bone growth, remodeling, and homeostasis is a topic of intense interest. ${ }^{4-6}$ miRNAs are small, non-coding RNAs that inhibit mRNA transcript translation and/or degrade transcripts through direct complementary binding. ${ }^{7}$ This mechanism of action allows miRNAs to negatively regulate gene expression. ${ }^{7}$ Of relevance to osteoporosis, the deletion of Dicer (the endoribonuclease that generates mature miRNAs) in mature osteoblasts has been shown to increase bone mass in adult mice, demonstrating that miRNAs in mature osteoblasts negatively regulate bone formation in the adult skeleton. ${ }^{8}$ This evidence suggests that targeting miRNA activity in mature osteoblasts may be a successful therapeutic strategy for age-related bone loss.

Therefore, here we chose to further investigate the mechanism(s) by which miRNAs negatively regulate osteoblastogenesis and bone formation in vitro and in vivo. We 
first conducted a miRNA microarray screen in adult bone marrow-derived MSCs during the proliferation-to-mineralization transition. This revealed profound downregulation of several miRNAs, most notably miR-451. We found that transfection of miR-451's antagomir stimulated pre-osteoblast differentiation into a more differentiated, mineralized phenotype and that this phenotype was associated with upregulated RUNX2, ALP, and COL1A1 protein expression. Furthermore, we found

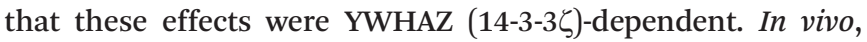
the treatment with miR-451's antagomir rescued trabecular bone loss in ovariectomized (OVX) mice and improved bone strength in both OVX and sham-operated mice. Ex vivo cultures of bone marrow-derived stromal cells from antagomirtreated mice displayed increased osteoblastogenesis and mineralization as well as upregulated Ywhaz, Runx2, Alp, and Col1a1 protein expression, validating our in vitro findings. On this basis, miR-451 appears to suppress osteoblastic differentiation, mineralization, and bone formation through a YWHAZ-mediated mechanism. Therefore, miR451 blockade may be a potential therapeutic strategy for senile osteoporosis.

\section{Materials and methods}

\section{Ethics statement}

All patient procedures were approved by the Ethics Committee of The Second Hospital of Jiaxing. All human subjects recruited for this study provided written informed consent prior to participation. All animal care and experimental procedures were approved by the Animal Ethics Committee of The Second Hospital of Jiaxing. The procedures followed in this study were in accordance with the standards set forth in the Guide for the Care and Use of Laboratory Animals (eighth edition, National Institutes of Health (NIH)).

\section{Human bone marrow collection and processing}

After obtaining written informed consent, bone marrow aspirates were provided by six healthy, non-medicated pre-menopausal female donors (age: 33-40 years). The bone marrow was processed to isolate mesenchymal stem cells (MSCs) using a Ficoll Histopaque density gradient (Sigma-Aldrich) and osteogenically differentiated in vitro as previously described with minor modifications. ${ }^{9}$ Briefly, cells were maintained in Alpha Modified Eagle's medium ( $\alpha$-MEM) supplemented with $10 \% \mathrm{FBS}$, penicillin $\left(100 \mathrm{U} \mathrm{ml}^{-1}\right)$, streptomycin (0.1 mg ml $\mathrm{m}^{-1}$ ), and $0.1 \mathrm{M}$ ascorbic acid-2 phosphate (all Sigma-Aldrich). After four days, non-adherent cells were removed, establishing time point 0 . The remaining adherent cells were cultured with fresh medium containing $1.0 \times 10^{-7}$ $\mathbf{M}$ dexamethasone (Sigma-Aldrich) to create the bone differentiation medium. After the first confluence, $10 \mathrm{mM}$ $\beta$-glycerophosphate (Sigma-Aldrich) was added to the medium to create the bone mineralization medium.

Cells were collected at time point 0 (proliferation time point), at the first confluence following addition of the bone differentiation medium (differentiation time point), and one week following addition of the mineralization medium (mineralization time point). Cell viability was measured using the Alamar Blue assay (Qcbio Science \& Technologies). The osteogenic phenotype was validated by alkaline phosphatase (ALP) activity (Sigma-Aldrich), mineral nodule formation, and osteocyte-specific transcript expression as described below. All cultures formed mineral nodules at the mineralization time point.

\section{ALP activity and Alizarin Red S assays}

The ALP activity was evaluated using a colorimetric ALP Activity assay kit (Abcam). Briefly, cells were added to a 96-well plate and the optical density (OD) was measured at $405 \mathrm{~nm}$ using a spectrometer. The ALP activity was then normalized to the DNA content using a Quant-iT PicoGreen kit (Invitrogen).

For Alizarin Red S staining, cells were fixed in $70 \%$ icecold ethanol for $1 \mathrm{~h}$. After rinsing with distilled water, cells were stained with $40 \mathrm{mM}$ Alizarin Red S (pH 4.2) for $10 \mathrm{~min}$ under gentle agitation. Alizarin Red $\mathrm{S}$ staining was captured by light microscopy.

The Alizarin Red S mineralization assay was performed by dissolving the stained deposits in $10 \%(\mathrm{v} / \mathrm{v})$ acetic acid for 30 min followed by $10 \%$ ammonium hydroxide (w/v) for $30 \mathrm{~min}$ to ensure a $\mathrm{pH}$ of 4.2 . OD was measured at $405 \mathrm{~nm}$ using a spectrometer. Mineralization was then normalized to DNA content using a Quant-iT PicoGreen kit (Invitrogen).

\section{miRNA microarray analysis}

A thorough miRNA microarray analysis was performed on the bone marrow-derived MSCs as previously described in order to determine the changes in miRNA expression over the various differentiation phases. ${ }^{9}$ Briefly, the total RNA was isolated using the TRIzol reagent (Life Technologies) from cells at the proliferation time point, the differentiation time point, and the mineralization time point. RNA integrity was confirmed by electrophoresis, and RNA concentration was calculated spectrophotometrically (NanoDrop ND-1000, Thermo Fisher Scientific). Human (hsa) miRNA expression profiling was conducted using miRCURY LNA microRNA Array version 11.0 (Exiqon). RNA samples were labeled with the miRCURY LNA microRNA Power Labeling Kit (Exiqon) and hybridized in a hybridization station. miRNA microarrays were scanned and analyzed with GenePix Pro version 6.0. Comparisons were made between miRNA expressions at the proliferation time point, the differentiation time point, and the mineralization time point.

\section{Real-time Taqman PCR for miRNA expression}

RT primers and TaqMan probes from TaqMan microRNA assays (Applied Biosystems) were used to quantify mature hsa-miRNA expression levels. GAPDH was used as an internal control. 


\section{Quantitative real-time PCR (qRT-PCR)}

The total RNA was extracted with the Trizol reagent (Life Technologies). The total RNA $(2 \mu \mathrm{g})$ was purified (Thermo Fisher scientific) and reverse-transcribed into cDNA with a PrimeScript II 1st Strand cDNA Synthesis kit (Takara Bio). qRT-PCR was performed using the PrimeScript RT Master Mix (Takara Bio) on an ABI 7500 Real-Time PCR system (Applied Biosystems) as follows: $95{ }^{\circ} \mathrm{C}$ for $10 \mathrm{~min}, 40$ cycles of 95 ${ }^{\circ} \mathrm{C}$ for $10 \mathrm{~s}$ each, and $60.5{ }^{\circ} \mathrm{C}$ for $30 \mathrm{~s}$. The primers are listed in Table 1. GAPDH was used as an internal control.

\section{Primary human calvarial pre-osteoblasts}

Primary human fetal calvarial pre-osteoblast cultures were prepared as previously described with minor modifications. ${ }^{10}$ Briefly, individual calvaria were surgically isolated from fetal skull tissue. The sutures were segregated, and any adherent tissue was cleaned off by gentle scrapping. The pooled calvaria were repeatedly digested with $0.1 \%$ collagenase $\mathrm{P}$ and $0.05 \%$ trypsin (15 min per digestion) to release the calvarial pre-osteoblasts. After throwing out the first digestion, cells were collected from the following four digestions. Pre-osteoblasts were cultured in $\alpha$-MEM supplemented with $10 \%$ FBS, penicillin $\left(100 \mathrm{U} \mathrm{ml}^{-1}\right)$, and streptomycin $\left(0.1 \mathrm{mg} \mathrm{ml}^{-1}\right)$ to reach $\sim 80 \%$ confluence prior to further experimentation.

\section{In vitro miRNA transfection into human pre-osteoblasts}

The miR-451 mimic (agomir), miR-451 inhibitor (antagomir),

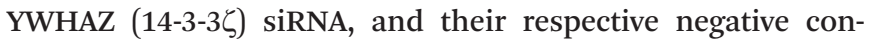
trols (miR-C or siCtrl) were all purchased from Dharmacon. Agomir and antagomir transfections were performed at 50$60 \%$ confluence with reduced-serum and antibiotic-free OptiMEM using 100 nM DharmFECT1 (Dharmacon). After six hours, the culture medium was replaced, and the cells were harvested at the indicated time points for later analyses.

\section{Western blotting}

Cells were lysed in ice-cold lysis buffer (Beyotime Institute of Biotechnology). Protein concentrations were measured with an Enhanced BCA Protein Assay Kit (Beyotime Institute of
Biotechnology). Equal amounts of total protein $(20 \mu \mathrm{g}$ per lane) were separated by $12 \%$ sodium dodecyl sulfatepolyacrylamide gel electrophoresis (SDS-PAGE) and then transferred onto a polyvinylidene fluoride (PVDF) membrane (Millipore). Membranes were then blocked for $1 \mathrm{~h}$ at room temperature with $5 \%$ bovine serum albumin (BSA, Sigma). Membranes were then incubated with the following primary antibodies overnight at room temperature (Cell Signaling Technologies unless otherwise stated): YWHAZ (diluted 1:1000, cat\# 8312), total Akt (diluted 1:1000, cat\# 4691), phospho-Akt ${ }^{\mathrm{Ser} 473}$ (diluted 1:1000, cat\# 9271), SMURF2 (diluted 1:1000, cat\# 12024), RUNX2 (diluted 1:1000, cat\# 12556), ALP (diluted 1: 10000, cat\# ab108337, Abcam), COL1A1 (diluted 1:1000, cat\# 84336), and GAPDH (diluted 1:1000, cat\# 2118). The blots were then incubated with species-appropriate horseradish peroxidase (HRP)-conjugated secondary antibodies (diluted 1: 1000, Cell Signaling Technologies) for $1 \mathrm{~h}$ at $37^{\circ} \mathrm{C}$ and finally developed using an enhanced chemiluminescence (ECL) reagent (Thermo Scientific). The ECL signals were captured and analyzed with the ChemiDoc MP imaging system and Image Lab version 4.0 (Bio-Rad).

\section{ALP-luciferase reporter assay}

HEK 293 cells were transfected with miR-C, miR-451 agomir, or miR-451 antagomir along with the ALP-luciferase (ALPLuc) reporter and a CMV promoter-driven $\beta$-galactosidase reporter (pCMV-bgal). The ALP-Luc reporter contains ALP's osteoblast-specific regulatory element. Luciferase activities were measured using a luciferase reporter assay kit (Promega) and a Glomax luminometer (Promega). Luciferase activity was normalized to $\beta$-galactosidase reporter activity.

\section{miR-451 target site prediction}

Putative targets for hsa-miR-451 were bioinformatically screened with TargetScan and miRanda using specific basepairing rules. ${ }^{11}$

\section{In vivo bone studies in mice}

Female BALB/c mice (weight: $18 \pm 5 \mathrm{~g}$ ) were housed in a $12 \mathrm{~h}$ light-dark cycle under controlled temperature $\left(22-24^{\circ} \mathrm{C}\right)$ and

Table 1 Primers used in qRT-PCR

\begin{tabular}{|c|c|c|}
\hline Gene & Forward primer & Reverse primer \\
\hline Human $B M P 2$ & 5'-ATG TTC GCC TGA AAC AGA GAC CCA-3' & 5'-CTT ACA GCT GGA CTT AAG GCG TTT C-3' \\
\hline Human SMAD1 & 5'-CCG CCT GCT TAC CTG CCT CCT GAA-3' & 5'-GAA CGC TTC GCC CAC ACG GTT GT-3' \\
\hline Human RUNX2 & 5'-TCT TCA CAA ATC CTC CCC-3' & $5^{\prime}$-TCT TCA CAA ATC CTC CCC-3' \\
\hline Human $A L P$ & 5'-ACC TCG TTG ACA CCT GGA AG-3' & 5'-CCA CCA TCT CGG AGA GTG AC-3' \\
\hline Human COL1A1 & 5'-GGG CAA GAC AGT GAT TGA ATA-3' & 5'-ACG TCG AAG CCG AAT TCC T-3' \\
\hline Hsa-miR-451 & 5'-AAA CCG TTA CCA TTA CTG AGT T-3' & Universal adaptor PCR primer (GeneCopoeia) \\
\hline Human GAPDH & 5'-CGA AGT CAA CGG ATT TGG TCG TAT-3' & 5'-AGC CTT CTC GGT GAA GAC-3' \\
\hline Murine Ywhaz & 5'-TGC AAC GAT GTA CTG TCT CTT TTG-3' & 5'-CGG TAG TCA CCC TTC ATT TTC A-3' \\
\hline Murine Runx2 & 5'-CCA GAA TGA TGG TGT TGA CG-3' & 5'-GGT TGC AAG ATC ATG ACT AGG G-3' \\
\hline Murine Alp & 5'-ATG GGC GTC TCC ACA GTA AC-3' & 5'-CTG AGT GGT GTT GCA TCG C-3' \\
\hline Murine Col1a1 & 5'-TGG CAA AGA CGG ACT CAA C-3' & 5'-GGC AGG AAG CTG AAG TCA TAA-3' \\
\hline Mmu-miR-451 & 5'-AAA CCG TTA CCA TTA CTG AGT T-3' & Universal adaptor PCR primer (GeneCopoeia) \\
\hline Murine Gapdh & 5'-GCC AAA AGG GTC ATC TC-3' & 5'-GTC TTC TGG GTG GCA GTG AT-3' \\
\hline
\end{tabular}


humidity (50-60\%) conditions. Mice were provided ad libitum access to standard chow and water. PBS (0.2 ml), miR-C (7 $\left.\mathrm{mg} \mathrm{kg}{ }^{-1}\right)$, or miR-451's antagomir $\left(7 \mathrm{mg} \mathrm{kg}^{-1}\right)$ were administered via anionic liposome (Invivofectamine 2.0, Life Technologies) into ovariectomized (OVX) or sham-operated mice for six weeks on day one through three during the first, third, and fifth weeks.

Micro-computed tomography (micro-CT) measurements were performed on a micro-CT scanner (InspeXio SMX225CT). Briefly, murine femora, tibiae, and vertebrae were scanned at an $18 \mathrm{~mm}$ nominal resolution, and 100 projections were acquired at an angular range of $180^{\circ}$. Imaging slices were reconstructed with a modified Feldkamp algorithm. ${ }^{12}$ The bone volume-to-tissue volume (BV/TV), trabecular number (Tb.N), and trabecular separation (Tb.Sp) were calculated using the mean intercept length method. ${ }^{13}$

Femoral bone formation was assessed through tetracycline injection (20 mg kg-1) into mice at 30-day time intervals. The mineralizing surface (MS), mineral apposition rate (MAR), and bone formation rate (BFR) were calculated using fluorescence imaging and Leica-Qwin software (Leica Microsystems).

For in vivo Alp and mineralization assays, bone marrow cells (BMCs) were flushed from murine femoral bone marrow into bone differentiation medium. BMCs were cultured in bone differentiation medium for 21 days prior to assay, with the medium replaced every $48 \mathrm{~h}$.

\section{Statistical analysis}

SPSS version 21.0 (SPSS) was employed for all statistical analyses. Data were reported as means and associated standard errors of the mean (SEMs) from experiments conducted in triplicate. Differentially-expressed miRNAs were determined using a paired $t$-test. Student's $t$-test was used for pairwise comparisons. One-way ANOVA and Newman-Keuls testing were applied for multiple comparisons. A $p$-value threshold of 0.05 was employed for all analyses.

\section{Results}

miRNA profiling across the proliferation-to-mineralization transition in adult human bone marrow-derived MSCs

We first studied adult human bone marrow-derived MSCs across the proliferation-to- mineralization transition, the process by which these MSCs differentiate into mature boneforming osteoblasts (Fig. 1A). Cultures of MSCs at the differentiation time point showed upregulated mRNA expression of several osteoblast genes, including BMP2, SMAD1, RUNX2, $A L P$, and COL1A1 ( $p<0.05$, Fig. 1B). miRNA microarray profiling revealed that 12 miRNAs were downregulated at the differentiation time point (Fig. 1C). We validated the downregulation of all 12 miRNAs by TaqMan miRNA PCR ( $p$ $<0.05$, Fig. 1D). Most notably, we observed a greater than 20fold downregulation in miR-451 at the differentiation time point (Fig. 1D). Furthermore, qRT-PCR of miR-451 expression revealed that miR-451 remained significantly downregulated throughout osteoblastic differentiation, from the differentia- tion time point through the mineralization time point $(p<$ 0.05 , Fig. 1E). On this basis, we selected miR-451 as a promising miRNA for further experimentation.

\section{miR-451 suppresses human osteoblastic differentiation}

We next studied the effects of transfecting miR-451's agomir or antagomir into primary human calvarial pre-osteoblasts. Stable transfection with miR-451's agomir or antagomir had no significant impact upon BMP2 secretion $(p>0.05$, Fig. 2A). However, miR-451's antagomir increased the ALP activity ( $p<0.05$, Fig. 2B), mineralization ( $p<0.05$, Fig. 2C), and mineralized nodule clone formation (Fig. 2D). In contrast, miR-451's agomir displayed the opposite effects on ALP activity and mineralization (Fig. 2B-D).

qRT-PCR revealed that miR-451's antagomir increased $A L P$ and COL1A1 mRNA expression, while the agomir displayed the opposite effects ( $p<0.05$, Fig. 2E). However, western blotting revealed that miR-451's antagomir increased RUNX2, ALP, and COL1A1 expression, while the agomir displayed the opposite effects ( $p<0.05$, Fig. $2 \mathrm{~F}$ ). This evidence indicates that miR-451's antagomir post-transcriptionally modulates RUNX2 expression.

As ALP is a direct target gene of the transcription factor RUNX2, ${ }^{14}$ we next utilized an ALP-luciferase promoter (ALPLuc) to determine whether miR-451's antagomir stimulates ALP promoter activity. We found that miR-451's antagomir stimulates ALP promoter activity, while the agomir displayed the opposite effects ( $p<0.05$, Fig. 2G). Taken together, miR451 appears to suppress human osteoblastic differentiation and mineralization.

\section{miR-451 suppresses YWHAZ expression in human pre-osteoblasts}

We applied bioinformatics analysis to predict miR-451's target gene(s) using the TargetScan and miRanda databases. The putative target gene was YWHAZ, which displayed a 7-nucleotide seed sequence match with miR-451 in its 3' UTR (Fig. 3A). Accordingly, miR-451's agomir repressed YWHAZ expression, whereas the antagomir derepressed YWHAZ expression (Fig. 3B). Western blotting validated these findings at the protein level (Fig. 3C). As previous work has reported that YWHAZ promotes AKT-mediated SMURF2 degradation, ${ }^{15}$ we also assessed the effects of miR-451's agomir and antagomir on AKT activation and SMURF2 protein expression. As postulated, we found that miR-451's agomir repressed AKTmediated SMURF2 degradation, whereas the antagomir derepressed AKT-mediated SMURF2 degradation (Fig. 3C).

Having established that miR-451 represses YWHAZ expression, we next investigated the effects of YWHAZ knockdown on osteoblastic differentiation. Silencing of YWHAZ by siRNA (Fig. 3D) had no significant impact on BMP2 secretion ( $p<$ 0.05, Fig. 3E) but did significantly decrease the ALP activity, mineralization, and mineralized nodule clone formation $(p<0.05$, Fig. 3F-H). Furthermore, qRT-PCR revealed that YWHAZ knockdown decreased $A L P$ and COL1A1 mRNA 
A

Mesenchymal stem cell (MSC)

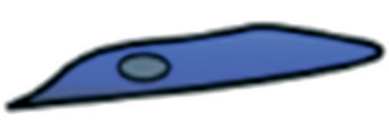

Proliferation timepoint (timepoint 0)
Pre-osteoblast

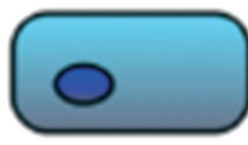

Differentiation timepoint
Mature osteoblast

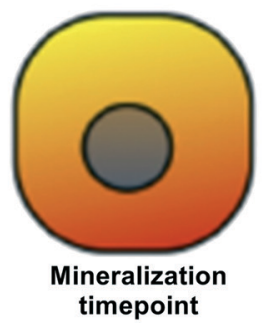

B

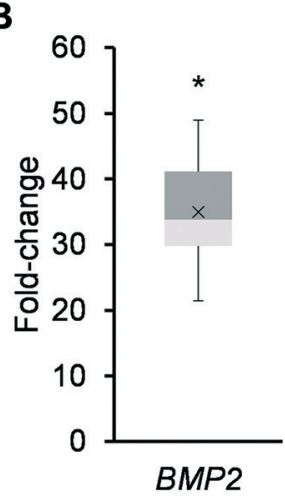

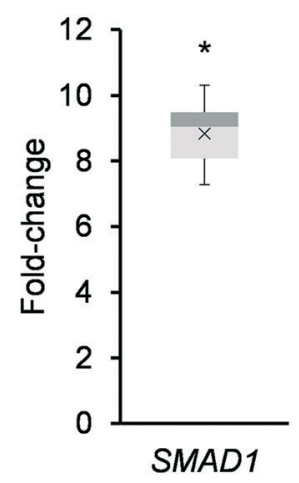

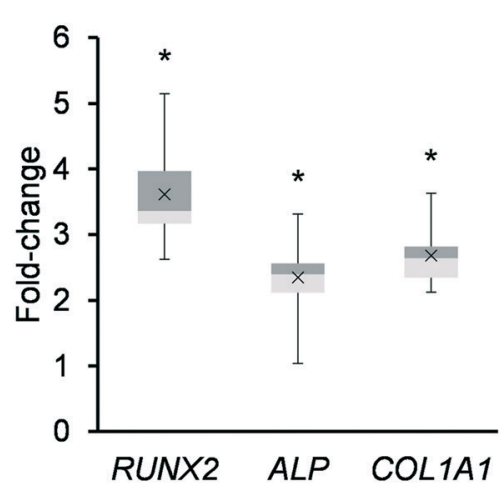

C

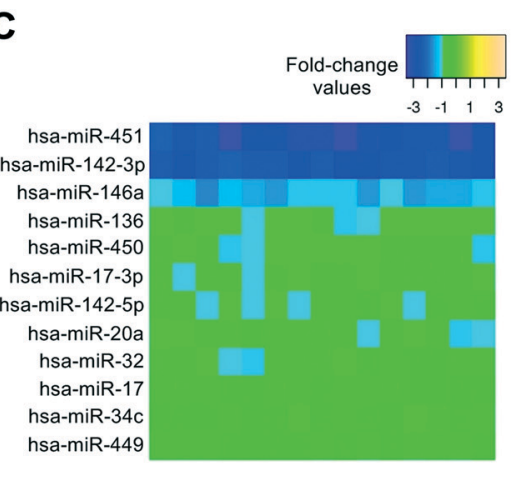

D

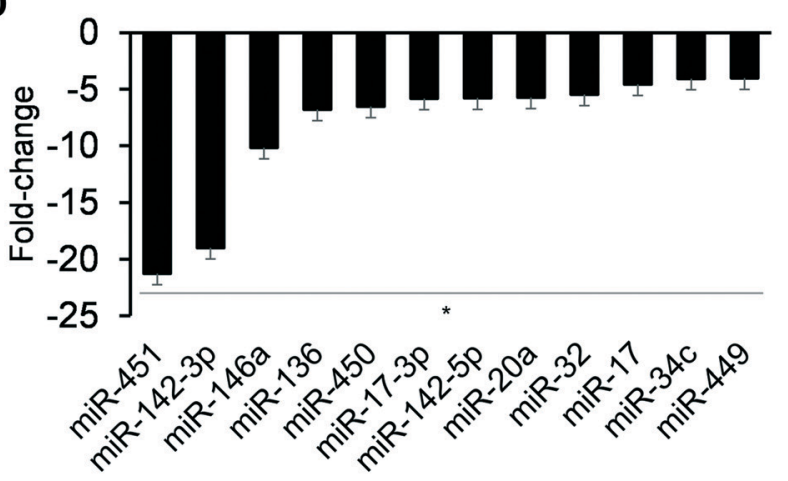

E

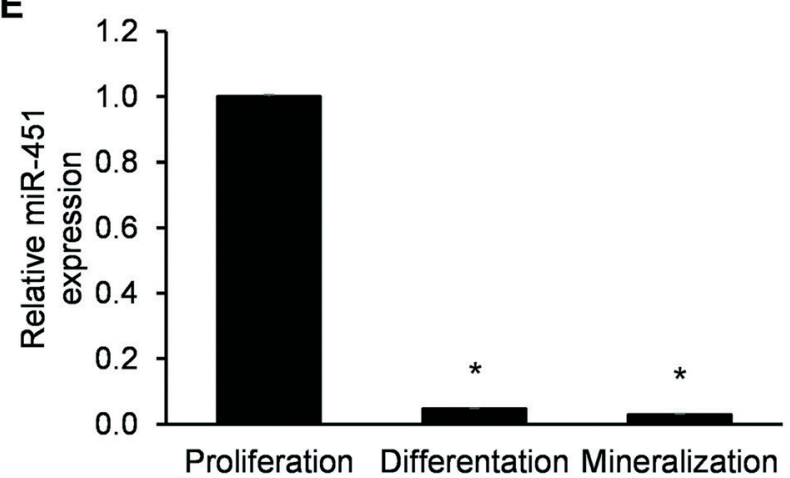

Fig. 1 miRNA profiling across the proliferation-to-mineralization transition in human adult bone marrow-derived MSCs. (A) Schematic overview of the osteogenic differentiation of adult bone marrow-derived MSCs. Sampling time points are indicated. (B) qRT-PCR-derived fold-changes in mRNA expression for the osteoblast genes BMP2, SMAD1, RUNX2, ALP, and COL1A1 at the differentiation time point relative to the proliferation time point (time point 0). (C) Heat map showing microarray expression of downregulated miRNAs at the differentiation time point relative to time point 0 . (C) TaqMan miRNA PCR validation of downregulated miRNAs. (D) qRT-PCR-derived expression of miR-451 at the proliferation time point (time point 0), differentiation time point, and mineralization time point. Data reported as means \pm SEMs. $* p<0.05$ versus proliferation time point (time point 0 ).

expression ( $p<0.05$, Fig. 3I). However, western blotting revealed that YWHAZ knockdown decreased RUNX2, ALP, and COL1A1 expression ( $p<0.05$, Fig. 3J). This evidence indicates that YWHAZ knockdown post-transcriptionally modulates RUNX2 expression.

As previous work has reported that AKT-mediated SMURF2 degradation promotes RUNX2 protein stability, ${ }^{15}$ we also assessed the effects of YWHAZ silencing on AKT activation and SMURF2 protein expression. As expected, we found that YWHAZ silencing repressed AKT-mediated SMURF2 degradation and upregulated SMURF2 levels (Fig. 3J). As SMURF2 degrades the RUNX2 protein, $^{15}$ upregulated SMURF2 explains the decreased RUNX2 protein levels ob- served (but unchanged RUNX2 mRNA levels) in siYWHAZ cells (Fig. 3I and J). Taken together, miR-451 suppresses YWHAZ/AKT-mediated RUNX2 protein stability by increasing SMURF2-mediated degradation of RUNX2, thereby inhibiting osteoblastic differentiation (Fig. 3K).

miR-451's antagomir reverses OVX-induced bone loss and improves bone strength in mice

To examine the anabolic effects of miR-451's antagomir in vivo, we injected OVX or sham-operated mice with the antagomir, miR-C, or PBS over a period of six weeks. We first measured miR-451 expression in bone extracts to validate 
A

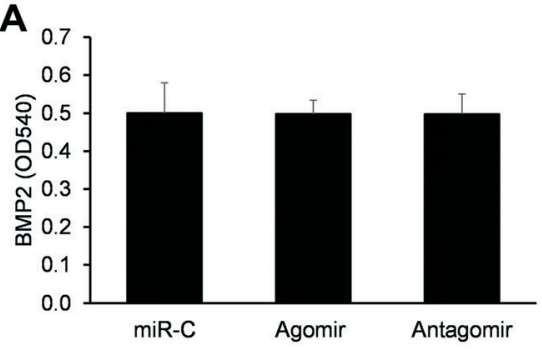

D

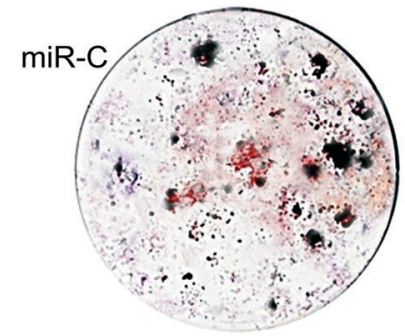

E

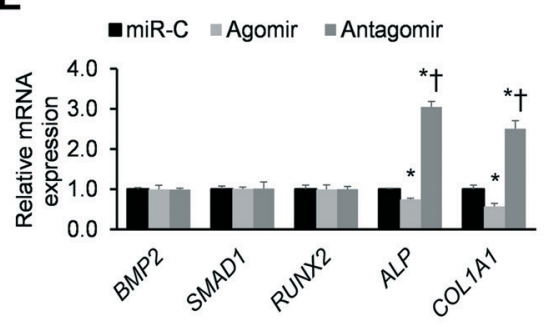

B

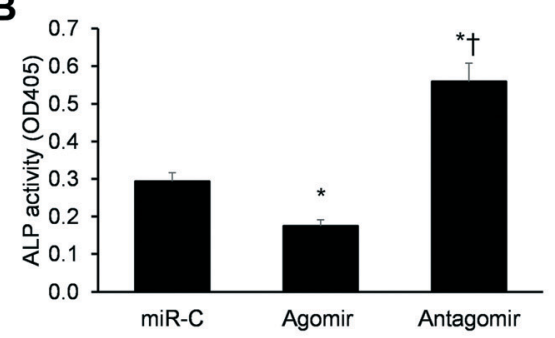

C

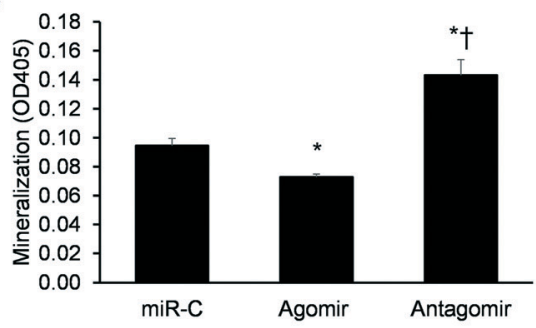

Fig. 2 miR-451 suppresses human osteoblastic differentiation. (A-C) Effects of the agomir or antagomir of miR-451 on pre-osteoblastic (A) BMP2 secretion (ELISA), (B) ALP activity, and (C) mineralization. (D) Representative images of alizarin-positive clone formation in pre-osteoblastic cultures. (E) qRT-PCR-derived mRNA expression of the osteoblast genes BMP2, SMAD1, RUNX2, ALP, and COL1A1. (F) Western blotting analyses of RUNX2, ALP, and COL1A1 protein expression. (G) Effects of the agomir or antagomir of miR-451 on ALP promoter activity using an ALP-luciferase (ALPLuc) reporter assay. Data reported as means \pm SEMs. ${ }^{*} p<0.05$ versus miR-C, $\dagger p<0.05$ versus agomir.

effective bone tissue delivery of the miR constructs $(p<0.05$, Fig. 4A). We then assessed trabecular bone at the femoral metaphysis, the tibial metaphysis, and the vertebral column (Fig. 4B). As expected, OVX produced significant trabecular loss, as evidenced by reduced BV/TV, reduced Tb.N, and increased Tb.Sp at all three bone sites $(p<0.05$, Fig. 4C and D, G-L). These destructive effects were partially rescued by miR-451's antagomir $(p<0.05)$, while PBS and miR-C showed no significant effects $(p>0.05)$. Notably, miR451's antagomir also increased the femoral strength and stiffness in both OVX and sham-operated mice $(p<0.05$, Fig. $4 \mathrm{E}$ and $\mathrm{F}$ ). Consistent with the increase in femoral Tb.N by miR-451's antagomir (Fig. 4D), the anabolic effects of the antagomir were validated by significant increases in the femoral bone formation parameters MAR and BFR (Fig. $4 \mathrm{~N}$ and $\mathrm{O}$ ). Taken together, miR-451's antagomir appears to reverse OVXinduced bone loss and improve bone strength in mice.

miR-451's antagomir suppresses YWHAZ expression in vivo, thereby stimulating osteoblastogenesis in mice

To explore the mechanism underlying the antagomir's anabolic effects on bone, we next isolated stromal cells from murine bone marrow on week six. As expected, OVX produced reductions in Alp activity, mineralization, and mineralized nodule clone formation for these bone marrow stromal cells $(p<0.05$, Fig. 5A-C). These destructive effects were partially rescued by miR-451's antagomir $(p<0.05)$, while PBS and miR-C showed no significant effects $(p>$ 0.05). Consistent with our in vitro transfection findings (Fig. 3), bone marrow stromal cells from antagomir-treated mice displayed significant upregulation in Ywhaz, Alp, and Col1a1 mRNA expression (Fig. 5D-G), while western blotting revealed significant upregulation in Ywhaz, Runx2, Alp, and Col1a1 protein expression (Fig. 5H). Taken together, the anabolic effects of miR-451's antagomir appear to be mediated through de-repression of YWHAZ expression and ALP activation.

\section{Discussion}

miRNAs regulate a wide range of biological processes through negatively regulating mRNA translation. ${ }^{7}$ Due to the debilitating effects of senile osteoporosis, there has been increasing interest in elucidating the roles of miRNA-based regulation in 
A

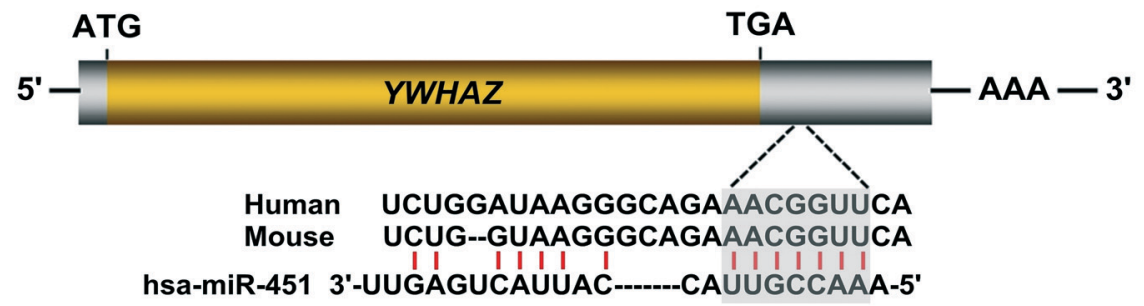

C

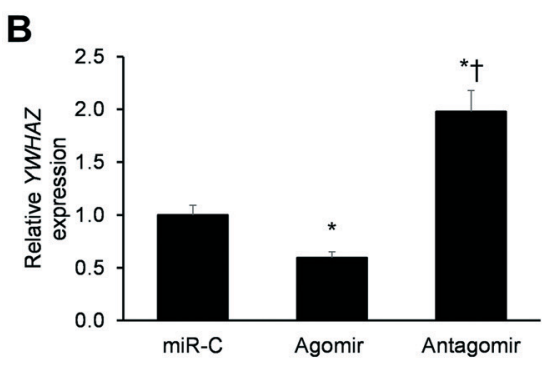

E

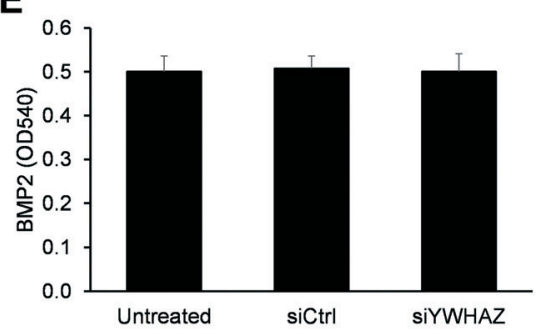

H

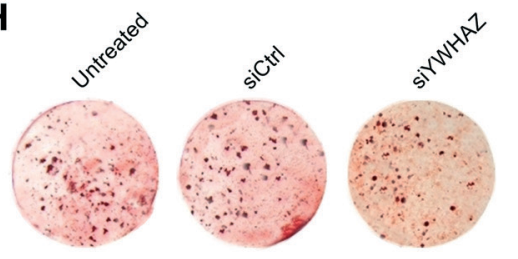

$\mathbf{F}$

I
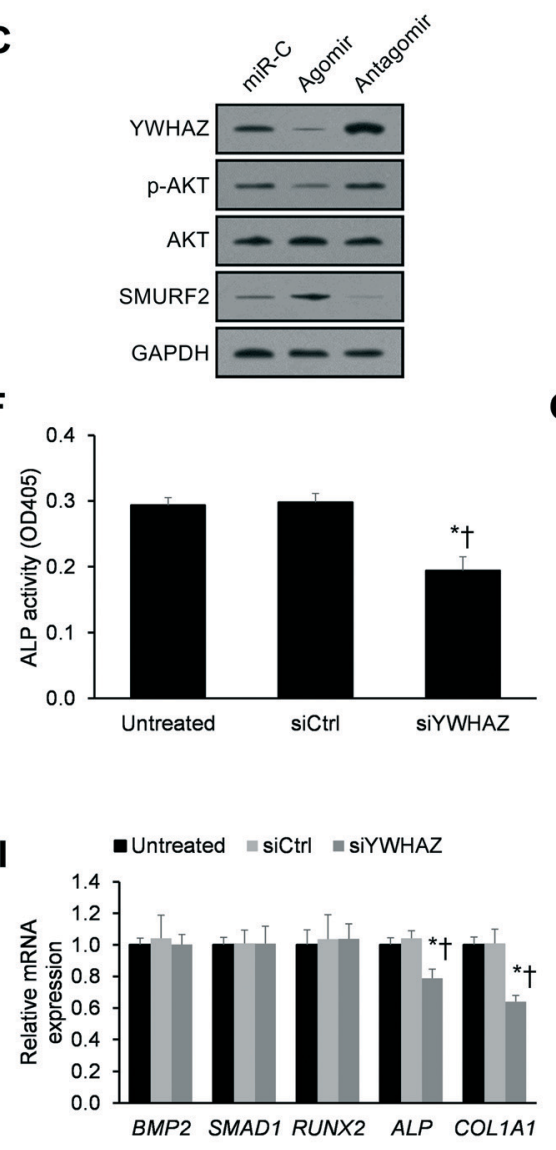

D

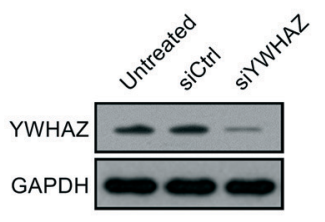

G
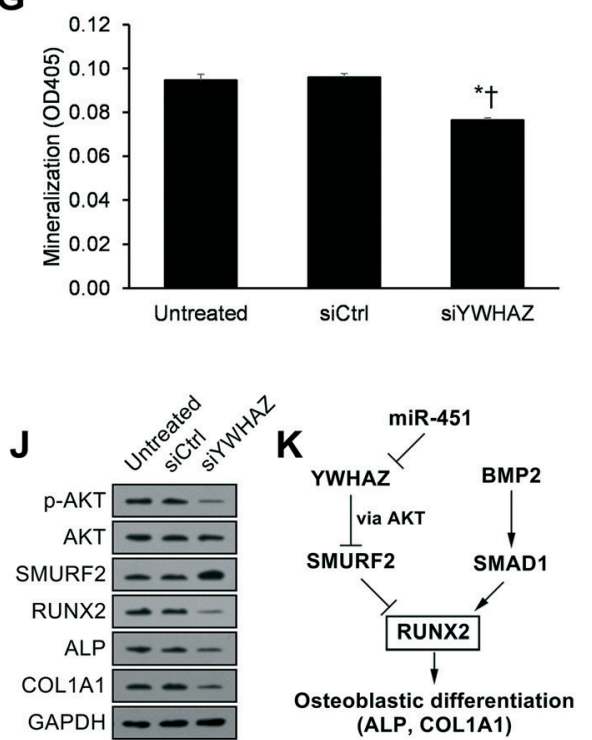

Fig. 3 miR-451 suppresses YWHAZ expression in human pre-osteoblasts. (A) Bioinformatic identification of miR-451's complementary binding to YWHAZ's 3'UTR. The 7-nucleotide seed sequence is highlighted in grey. (B) qRT-PCR-derived YWHAZ mRNA expression in pre-osteoblasts $24 \mathrm{~h}$

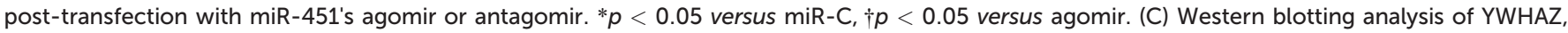
phospho-AKT, total AKT, and SMURF2 protein expression in pre-osteoblasts $24 \mathrm{~h}$ post-transfection with miR-451's agomir or antagomir. (D) YWHAZ protein expression in pre-osteoblasts transfected with scrambled control siRNA (siCtrl) or siRNA against YWHAZ (siYWHAZ). (E) BMP2 secretion, (F) ALP activity, (G) mineralization, and (H) alizarin-positive clone formation in pre-osteoblasts transfected with siCtrl or siYWHAZ. (I) qRTPCR-derived mRNA expression of the osteoblast genes BMP2, SMAD1, RUNX2, ALP, and COL1A1 and (J) western blotting analyses of phosphoAKT, total AKT, SMURF2, RUNX2, ALP, and COL1A1 protein expression in pre-osteoblasts transfected with siCtrl or siYWHAZ. (K) miR-451 suppresses YWHAZ, which results in SMURF2-mediated RUNX2 protein degradation and suppression of osteoblastic differentiation. Data reported as means \pm SEMs. ${ }^{*} p<0.05$ versus siCtrl, $\dagger p<0.05$ versus siYWHAZ.

adult bone growth, remodeling, and homeostasis. ${ }^{4-6}$ To date, several miRNAs have been directly associated with these processes, such as the promotion of osteoblastic differentiation (e.g., miR-29b, miR-196a, and miR-210) as well as the inhibition of osteoblastic differentiation (e.g., miR-23a-27a-24-2 cluster and miR-637). ${ }^{4}$

Our profiling of miRNA expression in adult bone marrowderived MSCs during the proliferation-to-mineralization transition, a critical period for adult osteoblastogenesis, ${ }^{16}$ re- vealed potent miR-451 downregulation. Zhang et al. have shown that miR-451 is significantly downregulated in human osteosarcoma tissues relative to matching non-cancerous tissue, with miR-451 negatively correlating with osteosarcoma metastasis and recurrence. ${ }^{17}$ Zhang et al. also demonstrated that artificial miR-451 expression suppresses osteosarcoma cell proliferation and invasiveness in vitro. ${ }^{17}$ Baglio et al.'s work revealed that miR-451 is significantly downregulated in osteogenically-differentiating bone marrow-derived MSCs. ${ }^{9}$ 

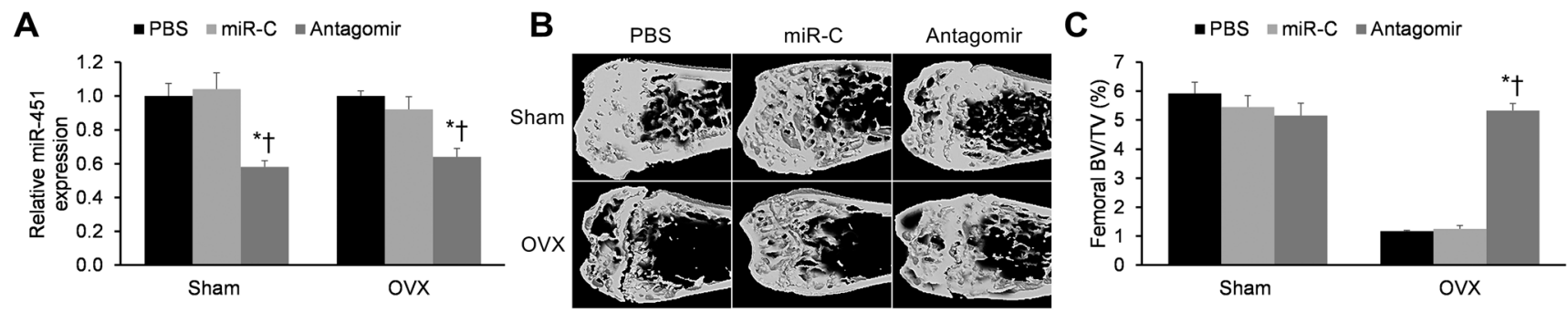

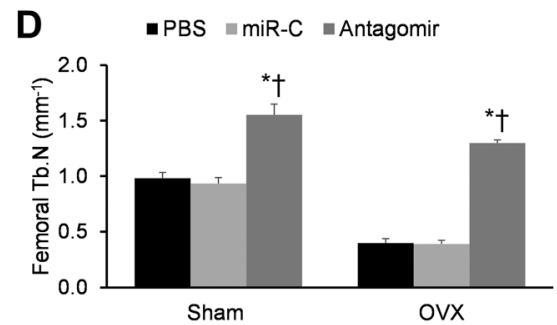

E
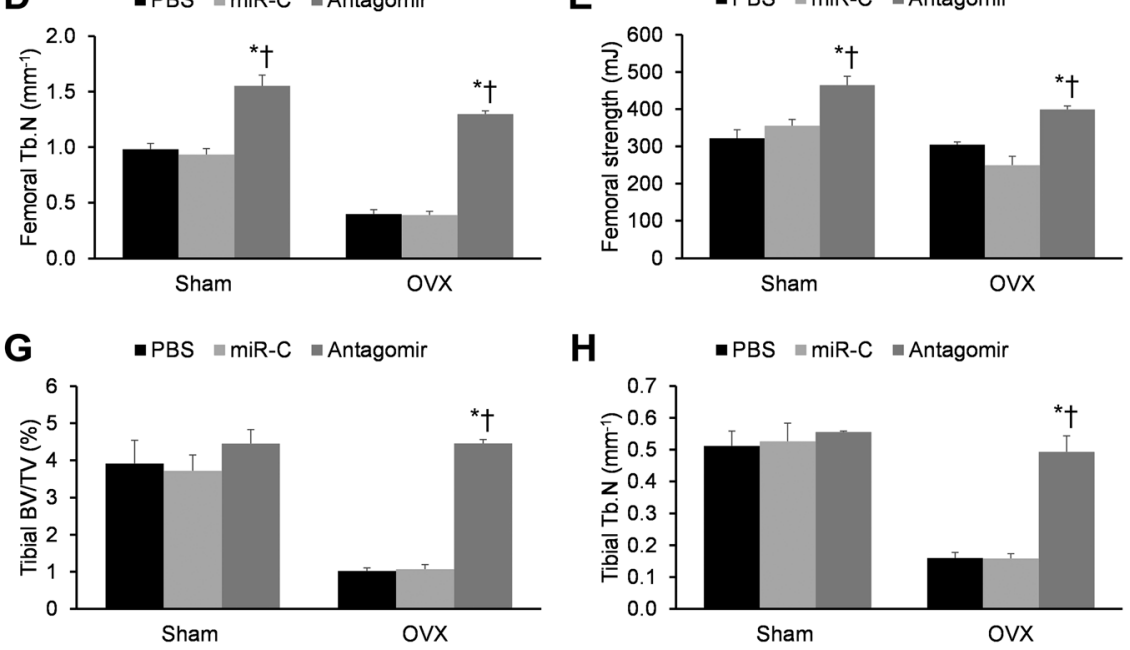

J
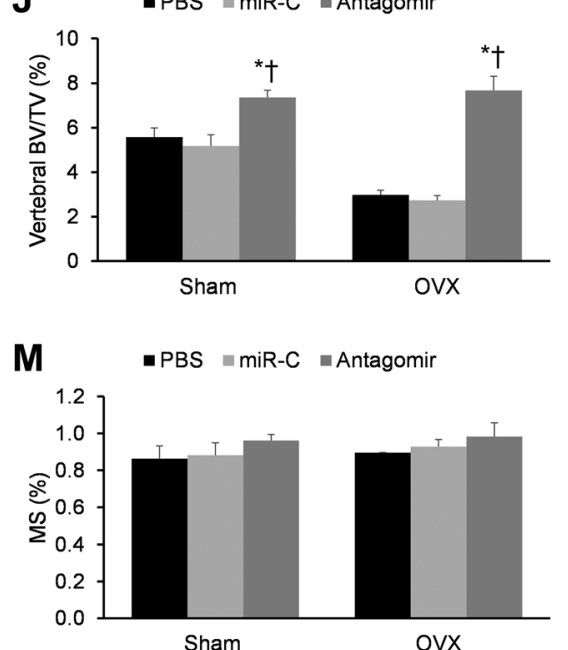

H

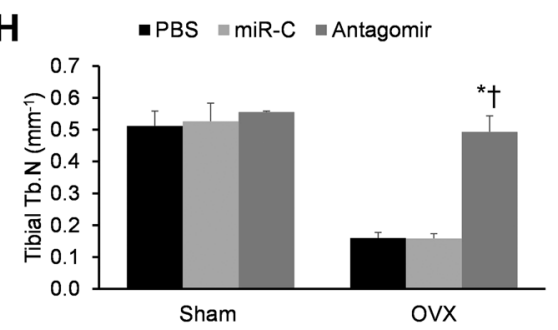

K

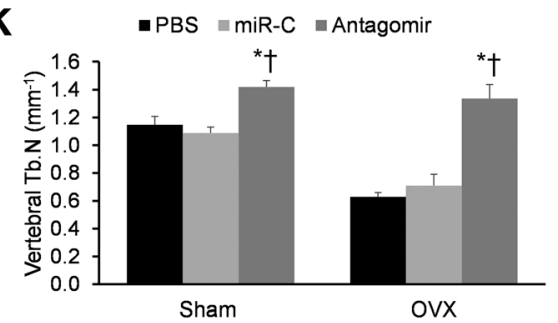

N

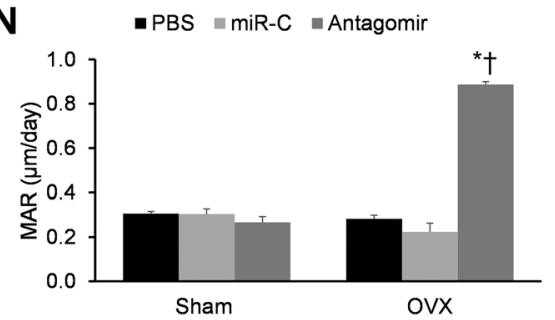

$\mathbf{F}$

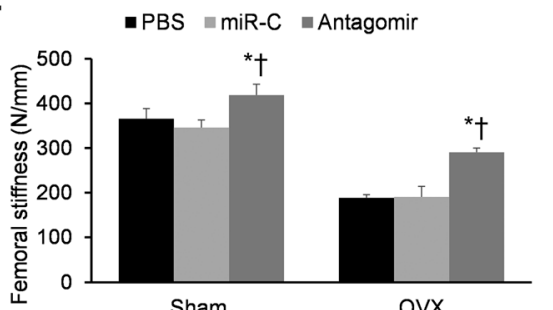

I

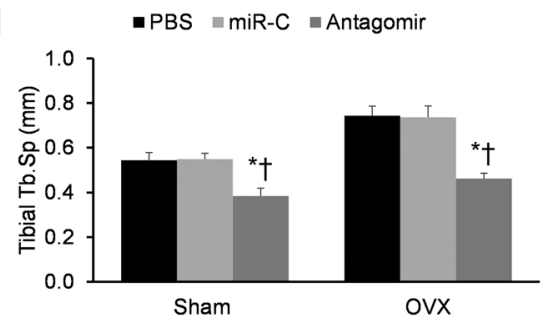

$\mathbf{L}$

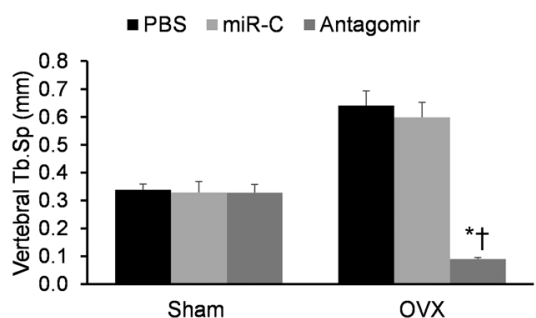

0

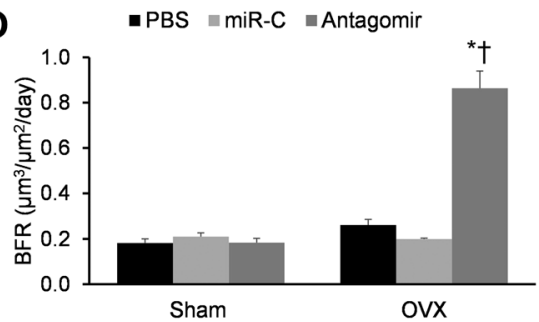

Fig. 4 miR-451's antagomir reverses ovariectomy (OVX)-induced bone loss and improves bone strength in mice. (A) Reduced miR-451 expression in bone following injection of its antagomir into six-week-old female Balb/c mice. (B) Representative micro-CT images of the femoral epiphyses in sham and OVX mice. (C-F) Measurement of the structural micro-CT parameters, including BV/TV, Tb.N, Tb.Sp, bone strength, and bone stiffness for (C-F) the femoral epiphyses, (G-l) tibial epiphyses, and (J-L) vertebrae. Femoral bone histomorphometric measures are also provided, including $(\mathrm{M})$ the mineralizing surface (MS), (N) mineral apposition rate (MAR), and (O) bone formation rate (BFR). $n=15$ mice/group. Data reported as means \pm SEMs. $* p<0.05$ versus matching PBS group, $\dagger p<0.05$ versus matching miR-C group.

This combined evidence suggests that miR-451 plays a key role in suppressing osteoblastogenesis.

Applying an antagomir approach here, we found that antimiR-451 stimulated pre-osteoblast differentiation into a more differentiated, mineralized phenotype in a YWHAZdependent manner and that this phenotype was associated with upregulated RUNX2, ALP, and COL1A1 protein expres- sion. Our ex vivo experiments on anti-miR-451-treated mice subjects mirrored these findings. Indeed, previously published evidence from other cell lines reveals that miR-451 directly suppresses YWHAZ transcription. ${ }^{18,19}$ In turn, Choi et al. demonstrated that YWHAZ activates Akt signaling in murine myoblasts, which increases RUNX2 protein stability by reducing SMURF2-mediated degradation of RUNX2. ${ }^{15}$ As 

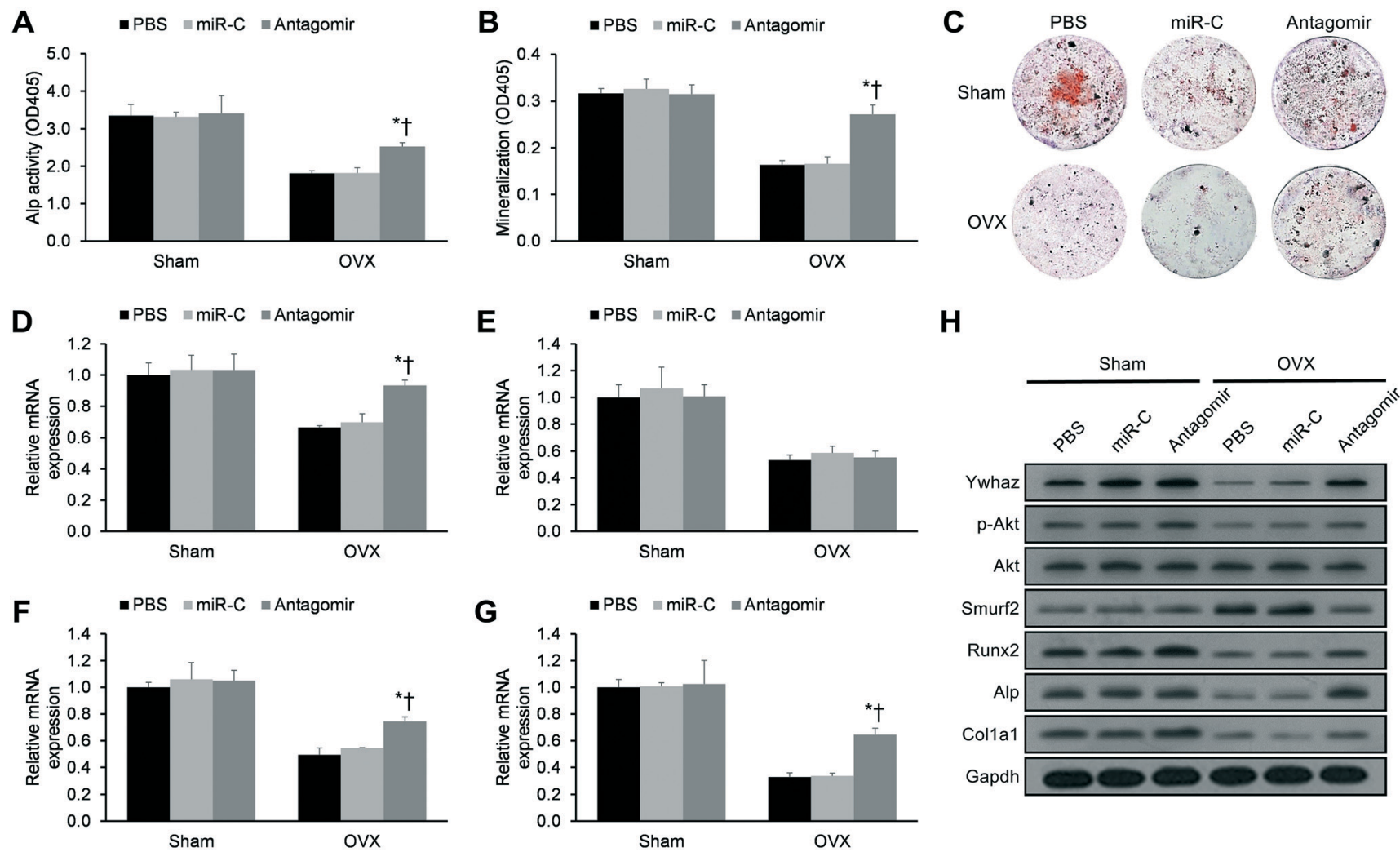

$$
\text { Sham OVX }
$$

Fig. 5 miR-451's antagomir suppresses YWHAZ expression in vivo, thereby stimulating osteoblastogenesis in mice. Bone marrow stromal cells were derived from sham-operated (sham) or ovariectomized (OVX) mice given PBS, scrambled miR (miR-C), or miR-451's antagomir. Measurements of (A) Alp activity and (B) mineralization are provided. (C) Representative images of alizarin-positive colony formation. (D-G) qRT-PCRderived mRNA expression levels of (D) Ywhaz, (E) Runx2, (F) Alp, and (G) Col1a1. (H) Western blotting analyses of murine Ywhaz, phospho-Akt, total Akt, Smurf2, Runx2, Alp, and Col1a1 protein expression. Data reported as means \pm SEMs. $* p<0.05$ versus matching PBS group, $\dagger p<0.05$ versus matching miR-C group.

RUNX2 is necessary for MSC commitment to the osteoblastic lineage, ${ }^{20}$ an alternative strategy to direct the inhibition of miR-451 is to target the downstream protein SMURF2 that degrades RUNX2. ${ }^{15}$ Although SMURF2 inhibitors are not currently available, ${ }^{21}$ the development of SMURF2 inhibitors may be a promising direction for promoting osteoblastic differentiation in senile osteoporosis patients.

Notably, we found that miR-451 had no significant impact upon BMP2 or SMAD1 expression in vitro. As BMP2 and SMAD1 lie upstream of AKT and RUNX2, ${ }^{13,15,22,23}$ BMP2 and SMAD1 expression would not be significantly affected by miR-451's regulation of YWHAZ/AKT-mediated RUNX2 protein stability.

In vivo, we found that anti-miR-451 rescued trabecular bone loss in OVX subjects and improved bone strength in both OVX and sham-operated subjects. Therefore, medicinal chemists should look into inhibition of miR-451 as a therapeutic strategy to promote osteoblastogenesis in senile osteoporosis patients. Although some known bioactive compounds (e.g., doxorubicin, docetaxel, vinca alkaloids) have been previously shown to regulate miR-451 expression, ${ }^{24}$ direct inhibition by either high-throughput-screened small molecules selectively targeting miR-451 biogenesis or synthetic antimiR-451 antagomirs may be more effective strategies in inhibiting production of mature miR-451. ${ }^{25}$
In conclusion, miR-451 suppresses osteoblastogenesis in vitro and in vivo. On this basis, miR-451 inhibition may serve as an effective anabolic therapeutic strategy in senile osteoporosis patients.

\section{Author contributions}

Conceived and designed the study: Jieen Pan, Chenglong Huang, Gang Chen, and Zhongwei Zhang. Performed the experimental procedures: Jieen Pan, Chenglong Huang, and Zhenhai Cai. Analyzed the data: Jieen Pan, Chenglong Huang, and Zhenhai Cai. Drafted the manuscript: Jieen Pan.

\section{Conflicts of interest}

The authors declare no competing interest.

\section{References}

1 P. J. Marie and M. Kassem, Eur. J. Endocrinol., 2011, 165, 1-10.

2 S. Khosla, J. A. Cauley, J. Compston, D. P. Kiel, C. Rosen, K. G. Saag and E. Shane, J. Bone Miner. Res., 2017, 32, 424-430.

3 A. Qaseem, M. A. Forciea, R. M. McLean and T. D. Denberg, Ann. Intern. Med., 2017, 166, 818-839. 
4 J. B. Lian, G. S. Stein, A. J. Van Wijnen, J. L. Stein, M. Q. Hassan, T. Gaur and Y. Zhang, Nat. Rev. Endocrinol., 2012, 8, 212.

5 L. De-Ugarte, G. Yoskovitz, S. Balcells, R. Güerri-Fernández, S. Martinez-Diaz, L. Mellibovsky, R. Urreizti, X. Nogués, D. Grinberg and N. García-Giralt, BMC Med. Genomics, 2016, 8, 75.

6 L. De-Ugarte, J. Serra-Vinardell, L. Nonell, S. Balcells, M. Arnal, X. Nogues, L. Mellibovsky, D. Grinberg, A. Diez-Perez and N. Garcia-Giralt, Hum. Cell, 2018, 31, 33-41.

7 J. A. Vidigal and A. Ventura, Trends Cell Biol., 2015, 25, 137-147.

8 T. Gaur, S. Hussain, R. Mudhasani, I. Parulkar, J. L. Colby, D. Frederick, B. E. Kream, A. J. Van Wijnen, J. L. Stein and G. S. Stein, Dev. Biol., 2010, 340, 10-21.

9 S. R. Baglìo, V. Devescovi, D. Granchi and N. Baldini, Gene, 2013, 527, 321-331.

10 P. Kushwaha, V. Khedgikar, J. Gautam, P. Dixit, R. Chillara, A. Verma, R. Thakur, D. Mishra, D. Singh and R. Maurya, Cell Death Dis., 2014, 5, e1422.

11 A. Grimson, K. K.-H. Farh, W. K. Johnston, P. Garrett-Engele, L. P. Lim and D. P. Bartel, Mol. Cell, 2007, 27, 91-105.

12 L. Feldkamp, L. Davis and J. Kress, J. Opt. Soc. Am. A, 1984, 1, 612-619.

13 V. Khedgikar, P. Kushwaha, J. Gautam, A. Verma, B. Changkija, A. Kumar, S. Sharma, G. Nagar, D. Singh and P. Trivedi, Cell Death Dis., 2013, 4, e778.
14 J.-j. Weng and Y. Su, Biochim. Biophys. Acta, Gen. Subj., 2013, 1830, 2839-2852.

15 Y. H. Choi, Y. J. Kim, H. M. Jeong, Y. H. Jin, C. Y. Yeo and K. Y. Lee, Rev. Geophys., 2014, 281, 3656-3666.

16 S. Zhou, J. S. Greenberger, M. W. Epperly, J. P. Goff, C. Adler, M. S. LeBoff and J. Glowacki, Aging Cell, 2008, 7, 335-343.

17 F. Zhang, W. Huang, M. Sheng and T. Liu, Tumor Biol., 2015, 36, 2041-2048.

18 W. Wang, L. Zhang, Y. Wang, Y. Ding, T. Chen, Y. Wang, H. Wang, Y. Li, K. Duan and S. Chen, Cell Death Dis., 2017, 8, e3071.

19 R. Su, J.-N. Gong, M.-T. Chen, L. Song, C. Shen, X.-H. Zhang, X.-L. Yin, H.-M. Ning, B. Liu and F. Wang, Oncotarget, 2016, 7, 77430 .

20 T. Komori, J. Cell. Biochem., 2005, 95, 445-453.

21 M. P. Smith, J. Ferguson, I. Arozarena, R. Hayward, R. Marais, A. Chapman, A. Hurlstone and C. Wellbrock, J. Natl. Cancer Inst., 2012, 105, 33-46.

22 N. Ghosh-Choudhury, S. L. Abboud, R. Nishimura, A. Celeste, L. Mahimainathan and G. G. Choudhury, J. Biol. Chem., 2003, 278, 16452-16452.

23 A. Mukherjee and P. Rotwein, J. Cell Sci., 2009, 122, 716-726.

24 S. Gezici and N. Sekeroglu, Cancer, 2017, 1, 4.

25 C. L. Haga, S. P. Velagapudi, J. L. Childs-Disney, J. Strivelli, M. D. Disney and D. G. Phinney, in Drug Target miRNA, Springer, 2017, pp. 179-198. 